Research Article

\title{
TfR mAb-Cross-Linked Rituximab/MTX-PEG-PLL-PLGA Drug-Loaded Nanoparticles Enhance Anticancer Action in B Lymphocytes
}

\author{
Ran Liu $\left(\mathbb{D},{ }^{1}\right.$ Gang Zhao, ${ }^{1}$ Shujun Wang, ${ }^{2}$ Yan Gu, ${ }^{1}$ Qi Han, ${ }^{1}$ and Baoan Chen $\mathbb{D}^{1}$ \\ ${ }^{1}$ Department of Hematology and Oncology, Zhongda Hospital Southeast University, Nanjing, Jiangsu Province 210009, China \\ ${ }^{2}$ Department of Blood Transfusion, Nanjing General Hospital of PLA, Nanjing, Jiangsu Province 210009, China \\ Correspondence should be addressed to Baoan Chen; cba8888@hotmail.com
}

Received 1 February 2019; Revised 16 June 2019; Accepted 29 July 2019; Published 11 September 2019

Guest Editor: Biaolin Peng

Copyright ( 2019 Ran Liu et al. This is an open access article distributed under the Creative Commons Attribution License, which permits unrestricted use, distribution, and reproduction in any medium, provided the original work is properly cited.

\begin{abstract}
Nanoparticles could enhance the drug targeted to the cancer cell by the enrichment of the drug levels, which leads to the improvement of the codelivery of both drugs for an antitumor effect. In the current study, we reported an efficient, local drugloaded delivery strategy with a nanoparticle-loaded system. After the synthesis of Rituximab/MTX-PEG-PLL-PLGA, the transferrin receptor monoantibody ( $\mathrm{TfR} \mathrm{mAb}$ ) was subsequently cross-linked to the nanoparticles. Compared to the traditional drug, the nanoparticle-loaded system can precisely and efficiently transport the Rituximab and Methotrexate (MTX) drug into SU-DHL-4 cells, a typical kind of B lymphocytes, which can significantly increase the cell apoptosis in the SU-DHL-4 cells. Thus, the multifunctional drug-loaded nanoparticles displayed the persistent stability and precise targeting properties, which enhanced the efficiency of anticancer efficiency in B lymphocytes.
\end{abstract}

\section{Introduction}

Although decades of efforts of research and billions of funding have enhanced our understanding of the underlying mechanisms of tumorigenesis, the death rate from cancer remains running at a high level nowadays [1]. Innovative strategies fighting cancer by inducing antitumor responses from the immune system are urgently needed $[2,3]$. Chemotherapeutics from the class of the anthracyclines have been shown to induce the death of cancer cells with immunogenic features and triggering immunogenic antitumor responses [4]. But they also bring big damage to the patient's immune system by the unspecific action of these cytotoxic drugs in systemic chemotherapy [5].

Nanotechnology carries the characteristics of antitumor drugs, and the emergence of nanotechnology has made it possible for drugs to be effectively delivered to tumors $[6,7]$. The single drug carrier can monitor the therapeutic effect in real time by combining radiographic imaging technology and antitumor intervention, and then the drug can be directly and efficiently reach the inside of the tumor, to maximize the reversal or overcome the antitumor multidrug resistance. Nanomedicine offers unique advantages in treating human cancers which are reported by several studies previously [8-10].

At present, the mechanism of tumor resistance is relatively complicated, but it also has shown that if a higher blood concentration is to be achieved and maintained, it is necessary to use a targeted site to deliver drugs to effectively control and reverse the starvation resistance of most tumor cells $[2,11]$. In order to better increase the accumulation of antitumor drugs in tumor sites, it is necessary to achieve nanocarrier preparation. There are three key points in the progress of nanocarrier preparation. Firstly, passive targeting of high permeability will allow a retention effect by enhancing the permeability [12]. Secondly, active tumor targeting of nanoparticles through the specific molecules on the surface of tumor cells can be an optimal strategy [13]. Thirdly, it should be damage-free or should give low toxicity to the surrounding of the normal cells. For the aim of having a damage-free tissue, it is necessary to apply responsive nanoparticles to achieve targets under the stimulation of 
physicochemical factors [14]. Some previous studies have found that the dendritic molecules have unique chemical structure properties, which make them act on tumors for a short period of time, inefficient tumor accumulation, and limited drug loading $[15,16]$. The novel nanodrug carrier shows excellent potentials in increasing the antitumor activity of the drug and reducing the side effects [17]. Although nanomedicine carriers are an important part of fighting cancer, cancer treatment drugs are also critical.

Rituximab was the first monoclonal antibody to be approved for the treatment of cancer, and it is estimated that more than 4 million people have been treated with Rituximab worldwide $[18,19]$. In the meantime, Methotrexate (MTX) is also still used in the treatment of a variety of tumors, including acute lymphocytic leukemia, breast cancer, osteosarcoma, primary central nervous system lymphoma, and head and neck cancers [20, 21]. Combined MTX and Rituximab with the nanodrug carrier may improve the accumulation of antitumor drugs and enhance permeability and retention effect.

Here, we aimed to investigate the effect of TfR mAbcross-linked Rituximab/MTX-PEG-PLL-PLGA drug-loaded nanoparticles, which served as a precise and efficient carrier for the Rituximab and Methotrexate (MTX) drug into SU-DHL-4 cells, a typical kind of B lymphocytes, which can significantly increase the cell apoptosis in the SUDHL-4 cells. The novel multifunctional drug-loaded nanoparticles persistently and precisely targeted SU-DHL-4 cells, which enhanced the efficiency of anticancer efficiency in B lymphocytes.

\section{Materials and Methods}

2.1. Synthesis of Rituximab/MTX-PEG-PLL-PLGA. The amount of PEG-PLL-PLGA was dissolved in $3 \mathrm{~mL}$ of a mixed solvent of dichloromethane-acetone-DMSO as the oil phase, while MTX (80 mg) (Sigma-Aldrich, St. Louis, Mo.) and Rituximab (40 mg) (Selleck Chemicals Houston, TX) were dissolved in $6 \mathrm{~mL}$ of $0.9 \%$ physiological saline as the aqueous phase. Then, the water phase was divided into 2 equal parts, labeled as aqueous phase 1 and aqueous phase 2, following the next two steps: (1) Aqueous phase 1 was poured into the oil phase, ultrasonically forming an emulsion, (2) and aqueous phase 2 was successively added dropwise to the oil phase, and the ultrasonication continued to form an emulsion. Finally, the above emulsion was added to $3 \mathrm{~mL}$ PVA $(3 \mathrm{mg} / \mathrm{mL})$ in $0.9 \%$ physiological saline. After ultrasonication for $6 \mathrm{~min}$, the solution was evaporated on a vacuum rotary evaporator to remove methylene chloride-acetone in the solution. The solution was centrifuged at $15000 \mathrm{r} / \mathrm{min}$ for $10 \mathrm{~min}$ twice in $3 \mathrm{~mL} 0.9 \%$ normal saline. The supernatant was collected and freeze-dried to solid. The product was obtained, weighed $(427.1 \mathrm{mg})$, and stored at $2-8^{\circ} \mathrm{C}$. In the preparation of PEG-PLL-PLGA nanoparticles, the procedure is the same as above using the $0.9 \%$ physiological saline without drugs.

To determine the content of Rituximab/MTX in micelles, a series of known amounts of Rituximab/MTX-loaded nanoparticles were separately dissolved in $3 \mathrm{~mL}$ PBS. A UV-Vis spectrophotometer (Cary 300, Agilent Technologies, USA) was used to determine the concentration of Rituximab at $278 \mathrm{~nm}$ and MTX at $258 \mathrm{~nm}$ to obtain a standard curve. Based on the standard curve, the drug loading content (DLC) and drug loading efficiency (DLE) could be calculated by the following equations:

$$
\text { DLC }(\%)=\frac{\text { weight of drug in nanoparticles }}{\text { weight of drug-loaded nanoparticle }} \times 100 \% \text {, }
$$

$$
\text { DLE }(\%)=\frac{\text { weight of drug in nanoparticles }}{\text { weight of feeding drug }} \times 100 \% \text {. }
$$

2.2. Preparation of TfR mAb/Rituximab/MTX-PEG-PLL$P L G A$. To prepare the TfR mAb-cross-linked Rituximab/MTX-PEG-PLL-PLGA, 30 mg Rituximab/MTX-PEGPLL-PLGA was dissolved in $3 \mathrm{~mL}$ PBS and ultrasonicated. Then, $300 \mu \mathrm{g}$ TfR mAb (Invitrogen) and $9 \mathrm{mg}$ Genipin were mixed gently at room temperature for 2 hours and an additional 12 hours at $2-8^{\circ} \mathrm{C}$. The solution was centrifuged at $15000 \mathrm{r} / \mathrm{min}$ for $10 \mathrm{~min}$ twice in $1 \mathrm{~mL} 0.9 \%$ normal saline. The product was obtained after lyophilization and stored at $2-8^{\circ} \mathrm{C}$.

To determine the TfR mAb modification rate in Rituximab/MTX-PEG-PLL-PLGA, a series of known amounts of TfR mAb-cross-linked Rituximab/MTX-PEG-PLL-PLGA nanoparticles were separately dissolved in $3 \mathrm{~mL}$ PBS. A $\mathrm{UV}-\mathrm{V}$ is spectrophotometer was used to determine the concentration of TfR $\mathrm{mAb}$ at $279 \mathrm{~nm}$. The modification rate (\%) was calculated by the above equation (1).

2.3. Characterization. The morphology of PEG-PLL-PLGA and drug-loaded nanoparticles was analyzed using a transmission electron microscope (TEM; Hitachi S-4800, Japan) with a voltage of $10 \mathrm{kV}$. The size and size distribution of nanoparticles were determined using a Malvern Zetasizer Nano ZS instrument (Malvern Instruments, Malvern, UK). A UV-Vis spectrophotometer (Cary 300, Agilent Technologies, USA) was used to determine the concentration of Rituximab, MTX, and TfR mAb.

2.4. In Vitro Drug Release. $300 \mathrm{mg}$ of the Rituximab/MTXloaded PEG-PLL-PLGA nanoparticles was dissolved in $5 \mathrm{~mL} 0.9 \%$ physiological saline. The diluted solutions were transferred into dialysis membrane tubes and immersed in $60 \mathrm{~mL}$ PBS $(0.01 \mathrm{M})$ then shaken at $150 \mathrm{rpm}$ at $37^{\circ} \mathrm{C}$. At scheduled intervals, $3 \mathrm{~mL}$ of solution was withdrawn from the release medium and replaced with the same volume of fresh PBS. The Rituximab/MTX content in the withdrawn solution was analyzed by a UV-Vis spectrophotometer and calculated based on the standard curve.

2.5. Cell Culture. The human SU-DHL-4 cell line was purchased from American Type Culture Collection (ATCC, Manassas, VA). The SU-DHL-4 cells were cultured in RPMI-1640 medium (Hyclone, UT, USA), supplied with $10 \%$ FBS (Gibco, CA, USA) and 1\% penicillin-streptomycin liquid at $5 \% \mathrm{CO}_{2}$ in a humidified incubator at $37^{\circ} \mathrm{C}$. 


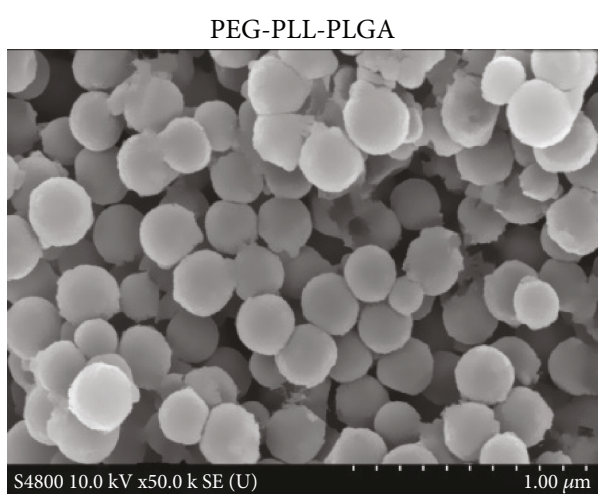

(a)

TfR mAb/Rituximab/MTXPEG-PLL-PLGA

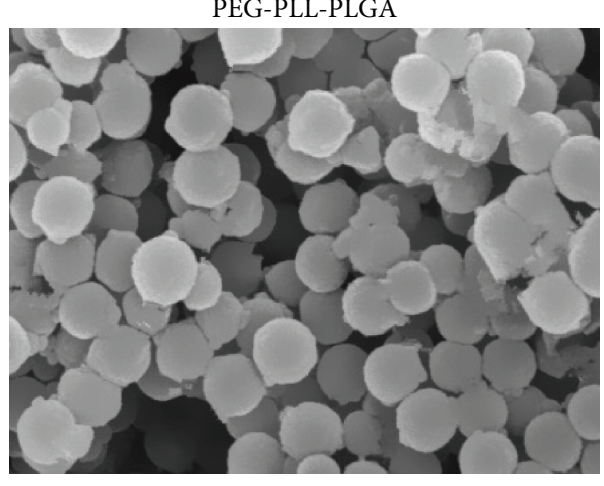

(c)

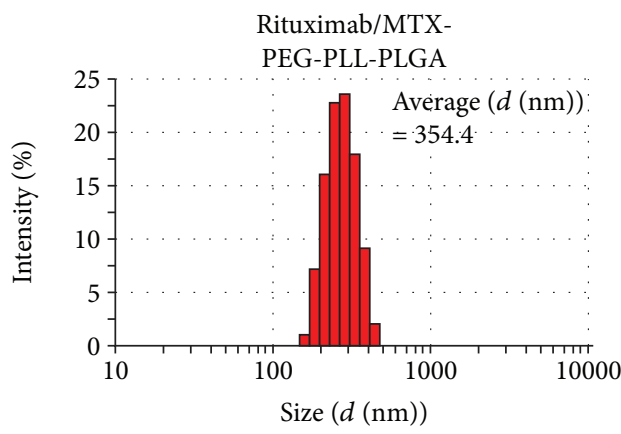

(e)

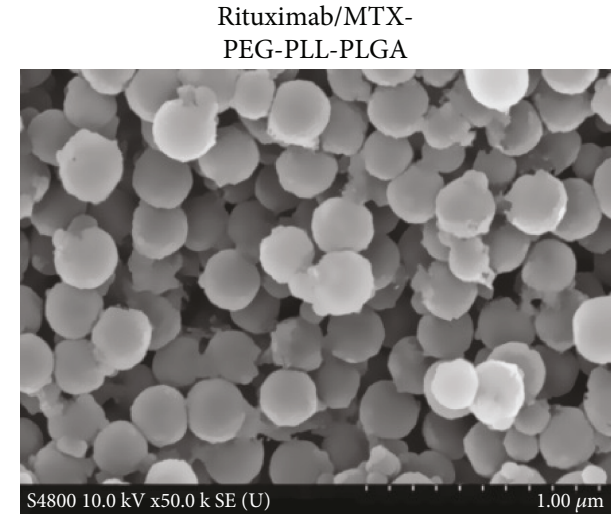

(b)

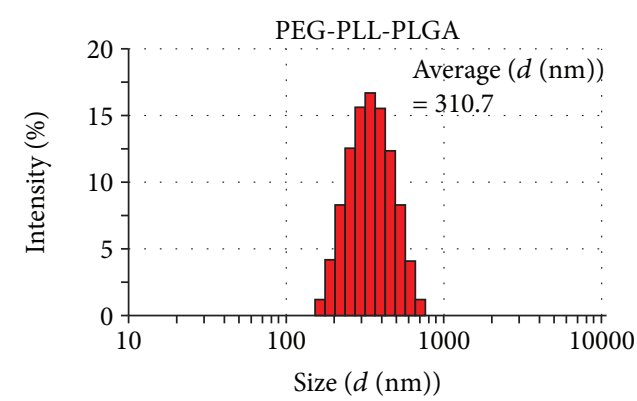

(d)

TfR mAb/Rituximab/MTXPEG-PLL-PLGA

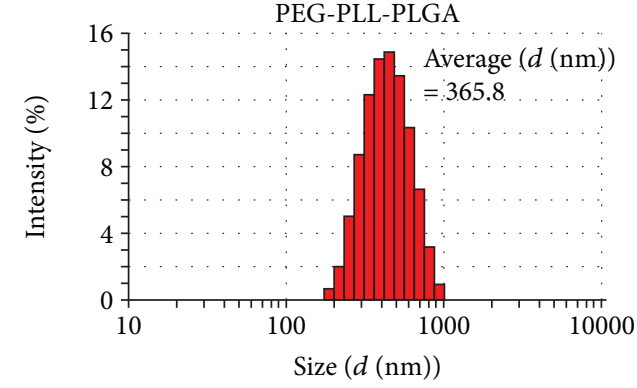

(f)

Figure 1: $(\mathrm{a}-\mathrm{c})$ The morphology of PEG-PLL-PLGA and drug-loaded nanoparticles was analyzed using TEM. Scale bar = $100 \mathrm{~nm}$. $(\mathrm{d}-\mathrm{f})$ Particle size and size distribution of nanoparticles were determined using a Malvern Zetasizer Nano ZS instrument. The average diameters $(d(\mathrm{~nm}))$ of particles were calculated by intensity.

2.6. In Vitro Cytotoxicity Evaluation. Cytotoxicity was measured using a CCK8 assay (Dojindo, Japan) according to the manufacturer's suggested procedures. SU-DHL-4 cells were seeded into 96-well plates at a concentration of $2.5 \times$ $10^{4}$ cells, and different concentrations of nanoparticles were added to be cultured for 48 and 72 hours, respectively. Then, $10 \mu \mathrm{L}$ of the CCK8 reagent was added to each well and incubated for 2 hours in the incubator. The OD value was measured at $450 \mathrm{~nm}$ with a microplate reader.
2.7. Analysis of Cell Cycle and Cell Apoptosis. SU-DHL-4 cells were inoculated into a 6-well plate at a concentration of $2 \times 10^{6}$ cells/well while the drug concentration was $175 \mu \mathrm{g} / \mathrm{L}$ Rituximab, $300 \mu \mathrm{g} / \mathrm{L}$ MTX, $175 \mu \mathrm{g} / \mathrm{L}$ Rituximab plus $300 \mu \mathrm{g} / \mathrm{L}$ MTX, and $2100 \mu \mathrm{g} / \mathrm{L}$ TfR mAb-MTX-PEG-PLLPLGA nanoparticles, and the medium treatment group was used as a control. After 48 hours of drug treatment, the cells were harvested and resuspended in $1 \mathrm{~mL}$ PBS. After centrifugation, the supernatant was discarded and the cells were 


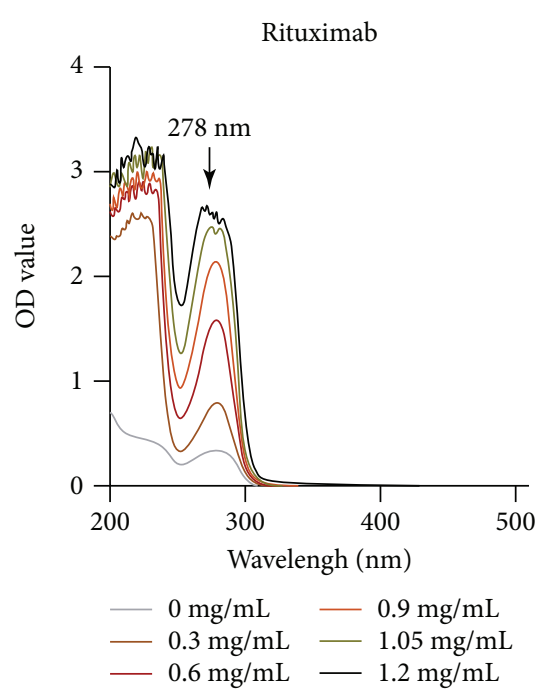

(a)

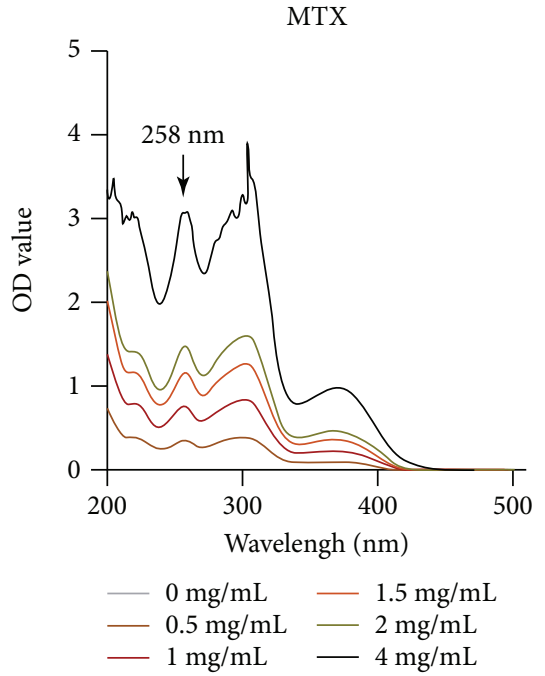

(b)

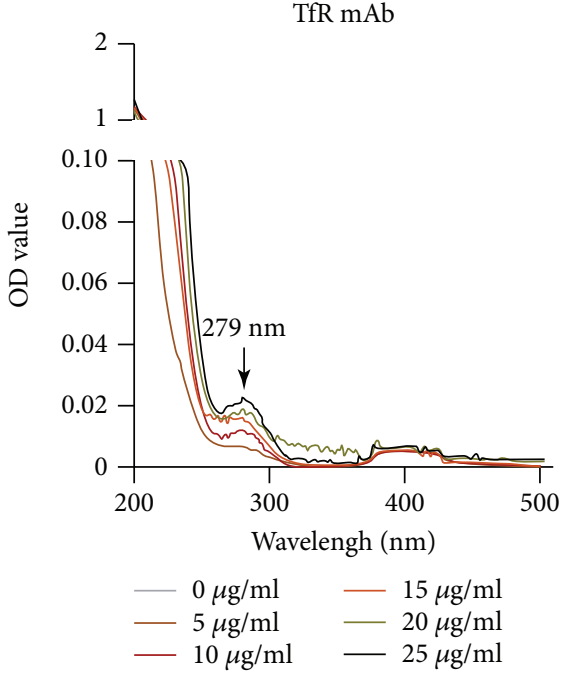

(c)

\begin{tabular}{lccc}
\hline Item & Rituximab & MTX & TfR mAb \\
\hline DLC (\%) & $8.17 \pm 0.02$ & $14.87 \pm 0.01$ & $/$ \\
DLE (\%) & $87.25 \pm 0.02$ & $79.41 \pm 0.01$ & $/$ \\
Modification rate (\%) & $/$ & $/$ & $70.90 \pm 2.33$
\end{tabular}

(d)

FIgURE 2: UVA sensitivity and stability of Rituximab- (a), MTX- (b), or TfR mAb- (c) loaded nanoparticles. (d) The drug loading content (DLC) and drug loading efficiency (DLE) of Rituximab and MTX and the modification rate of TfR mAb in TfR mAb/Rituximab/MTXPEG-PLL-PLGA nanoparticles.

resuspended in $0.5 \mathrm{~mL}$ PBS and $5 \mathrm{~mL}$ of precooled $70 \%$ ethanol. After being mixed well, the cells were fixed at $4^{\circ} \mathrm{C}$ overnight and then washed with PBS. RNase A $(50 \mu \mathrm{g} / \mathrm{mL})$ was added to dissolve for $30 \mathrm{~min}$ in a water bath at $37^{\circ} \mathrm{C}$, and then $5 \mu \mathrm{L}$ of PI $(50 \mu \mathrm{g} / \mathrm{mL})$ (Solarbio, China) was added to stain for $15 \mathrm{~min}$ at $4^{\circ} \mathrm{C}$ in the dark. Finally, the samples were detected by flow cytometry (BD Biosciences).

To detect cell apoptosis, cells were resuspended in $400 \mu \mathrm{L}$ of $1 \mathrm{x}$ Binding Buffer and supplied with $5 \mu \mathrm{L}$ FITC-Annexin V and $5 \mu \mathrm{L}$ PI $(50 \mu \mathrm{g} / \mathrm{mL})$ (Solarbio, China). The control cells were divided into the nonstained group, single-stained Annexin V group, single-stained PI group, and doublestained Annexin V plus PI group. The samples were gently bounced, mixed, and incubated for $15 \mathrm{~min}$ at room temperature in the dark. $400 \mu \mathrm{L}$ of $1 \mathrm{x}$ Binding Buffer was added to each tube and mixed. Finally, the samples were detected by flow cytometry (BD Biosciences) within 1 hour.

2.8. Statistics. Pharmacokinetic data were analyzed using the noncompartmental WinNonlin method. Statistical analysis was performed using the SPSS 22.0 program. $P$ values were obtained using the two-sample $t$-test. For cellular uptake, bidistribution, and antitumor activity data, statistical analyses were performed by ANOVA, with $P<0.05$ considered to be statistically significant and $P<0.01$ considered to be an extremely significant difference. Statistical analysis was performed using the GraphPad Prism 5 (GraphPad Software, San Diego, CA).

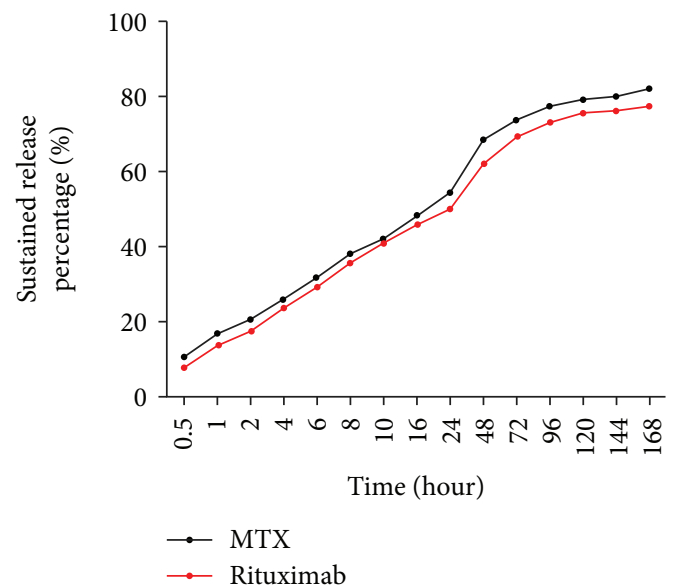

FIgURE 3: The sustained release percentage of MTX- and Rituximab-loaded nanoparticles in vitro.

\section{Results and Discussion}

Prior to carrying out efficacy studies, the Rituximab and MTX drug-loaded PEG-PLL-PLGA nanoparticles were synthesized, followed by cross-linking by TfR mAb. The particles of the drug-loaded nanoparticles were evaluated by transmission electron microscope (TEM) morphology, which showed uniform particle distribution in three kinds 


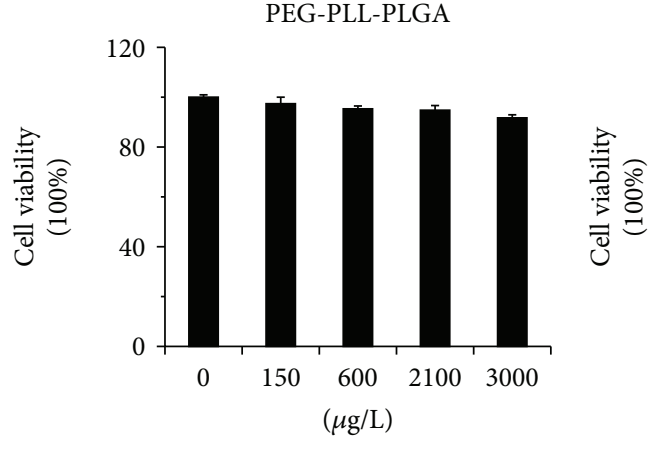

(a)

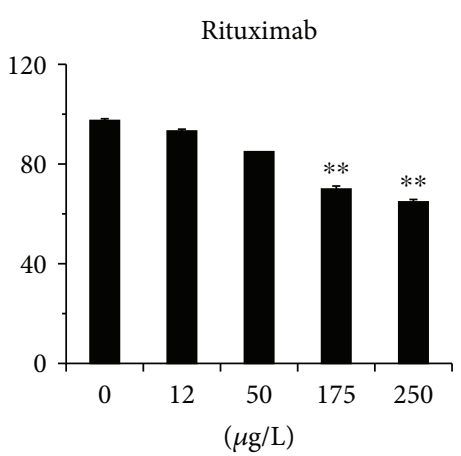

(b)

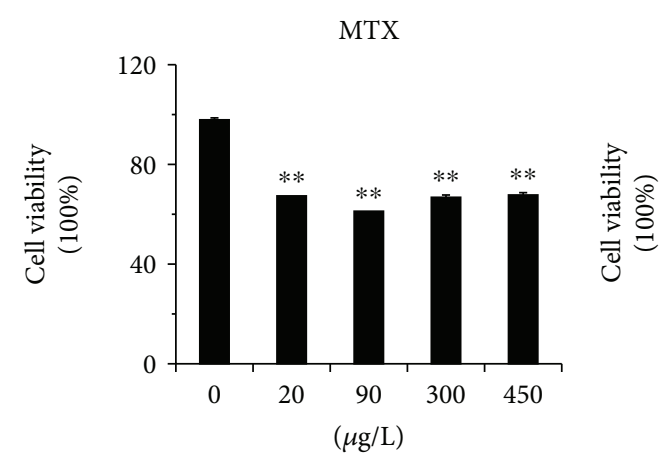

(c)

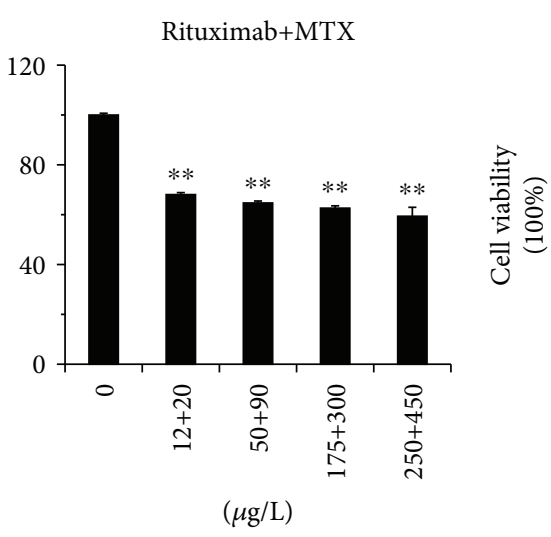

(d)

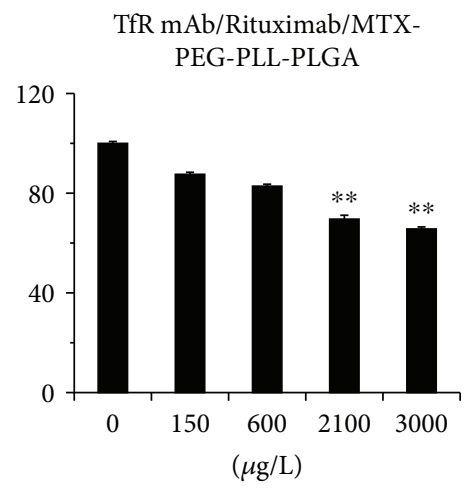

(e)

FIGURE 4: In vitro cytotoxicity evaluation of SU-DHL-4 cells using CCK assay. The SU-DHL-4 cells were treated by different drugs for 48 hours. (a) PEG-PLL-PLGA, (b) Rituximab, (c) MTX, (d) Rituximab combined with MTX, and (e) TfR mAb/Rituximab/MTX-PEGPLL-PLGA nanoparticles. Data are shown as the mean \pm SD. ${ }^{*} P<0.05 ;{ }^{* *} P<0.01$.

of nanoparticles before and after drug loading: PEG-PLLPLGA (Figure 1(a)), Rituximab/MTX-PEG-PLL-PLGA (Figure 1(b)), and TfR mAb Rituximab/MTX-PEG-PLLPLGA (Figure 1(c)). The size and size distribution of nanoparticles were also determined. The average diameter of the kinds of particles ranged from $310.7 \mathrm{~nm}$ before drug loading (Figure 1(d)) to $354.4 \mathrm{~nm}$ after Rituximab/MTX loading (Figure 1(e)) and to $365.8 \mathrm{~nm}$ after TfR mAb cross-linking (Figure 1(f)).

Then, a UV-Vis spectrophotometer was used to determine the concentrations of Rituximab at $278 \mathrm{~nm}$ (Figure 2(a)), MTX at $258 \mathrm{~nm}$ (Figure 2(b)), and TfR mAb at $279 \mathrm{~nm}$ (Figure 2(c)) in the drug-loaded nanoparticles. Based on the standard curve, the drug loading content (DLC) and drug loading efficiency (DLE) were calculated. The percentages of DLC of Rituximab and MTX were $8.17 \%$ and $14.87 \%$ (Figure 2(d)), respectively. The percentages of DLC of Rituximab and MTX were $87.25 \%$ and $79.41 \%$ (Figure 2(d)), respectively. In addition, the TfR mAb modification rate is up to $70.90 \%$ (Figure 2(d)), suggesting that the rates of drug loading and encapsulation were comparable and efficient.

The drug release of the nanoparticles in vitro was further evaluated. The sustained release rates of Rituximab and MTX reached $49.9 \%$ and $54.12 \%$, respectively (Figure 3 ). Until 168 hours ( 7 days), the sustained release efficiency was maintained to $77.6 \%$ and $81.81 \%$ (Figure 3), indicating that the sustained release of the two drugs in the nanoparticles owned a long effect.

To assess the cytotoxicity of the drugs, we treated the $\mathrm{B}$ lymphocytes and SU-DHL-4 cells by different concentrations of drugs (PEG-PLL-PLGA, Rituximab, MTX, Rituximab + MTX, and TfR mAb/Rituximab/MTX-PEG-PLL-PLGA), respectively. The cell viability of SU-DHL-4 cells was not affected treated with PEG-PLL-PLGA for 48 hours (Figure 4(a)), while it was significantly reduced treated with 175 and $250 \mu \mathrm{g} / \mathrm{L}$ Rituximab for 48 hours (Figure 4(b)), MTX (ranged from 20 to $450 \mu \mathrm{g} / \mathrm{L}$ ) for 48 hours (Figure 4(c)), and Rituximab combined with MTX for 48 hours (Figure 4(d)), respectively. Moreover, the cell viability of SU-DHL-4 cells treated with a high concentration of TfR mAb/Rituximab/ MTX-PEG-PLL-PLGA for 48 hours was significantly reduced, ranging from 0.59 to approximately $0.62(P<0.01$ vs. mock control), which was commensurate with other groups (Figure 4(e)).

We selected drug concentrations in subsequent experiments for further evaluation as follows: $175 \mu \mathrm{g} / \mathrm{L}$ Rituximab, $300 \mu \mathrm{g} / \mathrm{L}$ MTX, $175 \mu \mathrm{g} / \mathrm{L}$ Rituximab+300 $\mu \mathrm{g} / \mathrm{L}$ MTX, and $2100 \mu \mathrm{g} / \mathrm{L}$ TfR mAb/Rituximab/MTX-PEG-PLL-PLGA for 48 hours of treatment. The cell cycle was detected by flow cytometry (Figure 5(a)). Compared with the control group, Rituximab, MTX, or coprocessing of Rituximab plus MTX treatment resulted in a decrease in the proportion of $S$ phase 


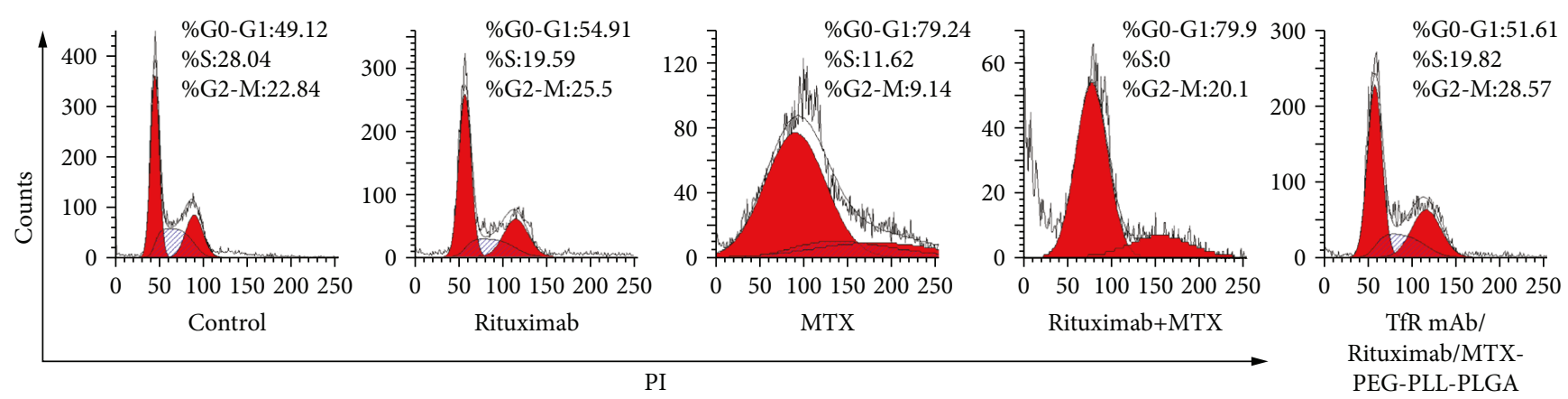

(a)

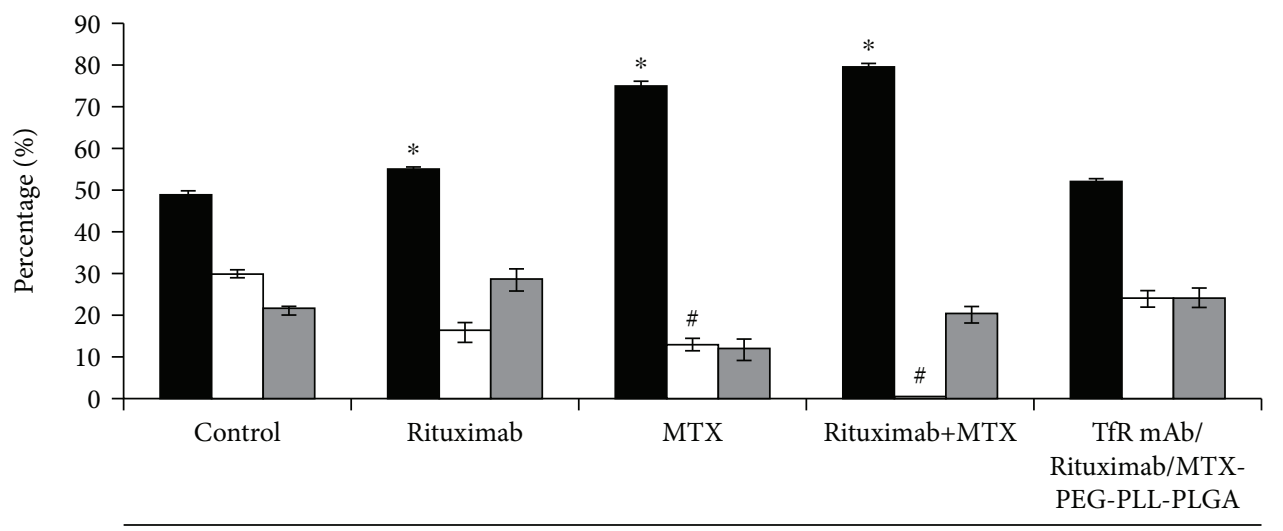

Treatment

\%G0-G1
$\square \mathrm{S}$
$\square \mathrm{G} 2-\mathrm{M}$

Figure 5: (a) Cell cycle analysis of SU-DHL-4 cells treated by different kinds of drug for 48 hours using flow cytometry. (b) The percentages of phases in the cell cycle are shown as the mean $\pm \mathrm{SD} .{ }^{*} P<0.05 ;{ }^{* *} P<0.01$.

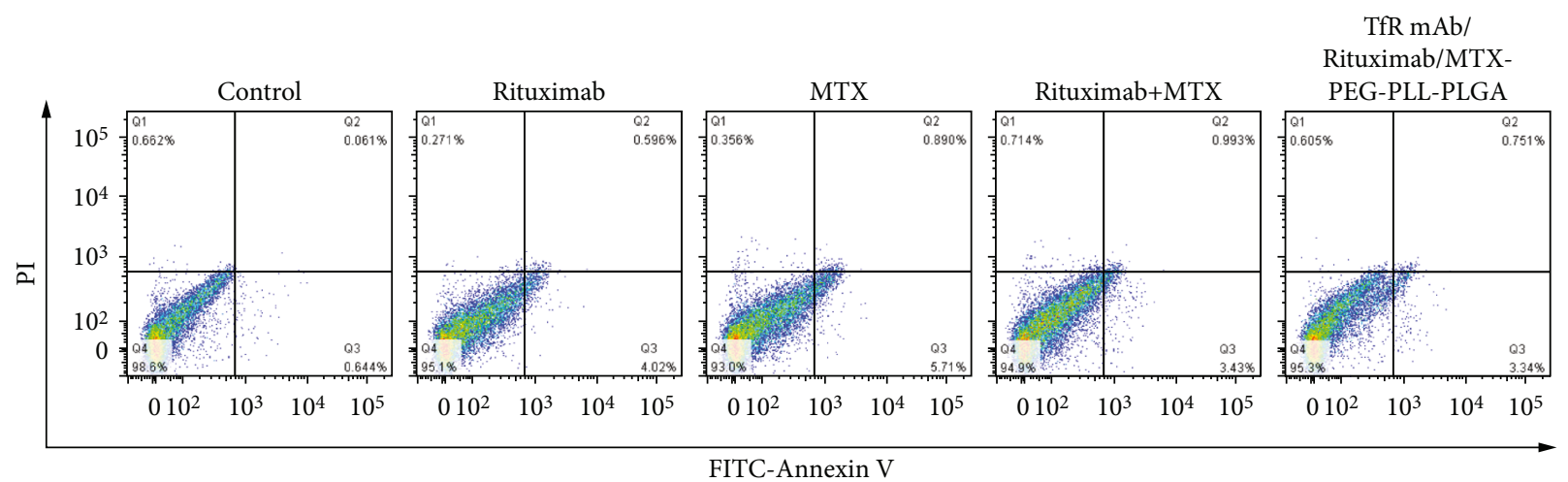

FIgURe 6: Cell apoptosis analysis of SU-DHL-4 cells treated by different kinds of drug for 48 hours using flow cytometry with PI and FITC-Annexin V staining.

cells while there is an increase in the proportion of G0-G1 (Figure 5(b)). However, TfR mAb/Rituximab/MTX-PEGPLL-PLGA treatment did not have a significant effect on the cell cycle (Figure 5(b)).

To further identify the cytotoxicity of the drugs, cell apoptosis affected by drugs was determinate by forward scatter
(FSC) and side scatter (SSC) in flow cytometry. Being measured for cell size and granularity in cell apoptosis, the cell shrinks and releases apoptotic blebs as reflected by decreasing FSC and increasing SSC compared to normal cells. Exposed phosphatidylserine can be detected in the presence of calcium using FITC-Annexin V. After SU-DHL-4 cells 
were treated with Rituximab, MTX, Rituximab+MTX, or TfR $\mathrm{mAb} /$ Rituximab/MTX-PEG-PLL-PLGA, the cell apoptosis rates of all treated groups were significantly higher than that of the control group (Figure 6). These results demonstrated the effect of cytotoxicity by TfR mAb/Rituximab/MTXPEG-PLL-PLGA drug-loaded nanoparticles which relied on cell apoptosis.

\section{Conclusions}

In summary, we showed novel anticancer effects of TfR mAb cross-linked Rituximab and MTX drug-loaded nanoparticles on B lymphocytes and preliminarily studied the interference mechanism involved in cell cycle and cell apoptosis. The multifunctional single drug-loaded nanoparticles displayed maintained stability and precise targeting, which enhanced the anticancer efficiency of cell death for B lymphocytes. This enhanced cancer treatment provides a potential strategy in clinical anticancer therapy and deeper insights into the physiological and pathophysiological variability among individual patients. These findings also guide the design of precise nanomedicine towards personalized cancer treatments.

\section{Data Availability}

The data used to support the findings of this study are included within the article.

\section{Conflicts of Interest}

The authors declare that there is no conflict of interest regarding the publication of this article.

\section{Acknowledgments}

This work was supported by the National Natural Science Foundation of China Program (81600161).

\section{Supplementary Materials}

A precise and efficient carrier for the Rituximab and Methotrexate (MTX) was designed as nanomedicine for anticancer action in B lymphocytes. After the synthesis of Rituximab/MTX-PEG-PLL-PLGA, the TfR mAb was subsequently cross-linked to the nanoparticles. The nanoparticleloaded system can precisely and efficiently transport the Rituximab and MTX drug with long-term sustained release of into SU-DHL-4 cells. The TfR mAb/Rituximab/MTXPEG-PLL-PLGA nanoparticle increased the cell apoptosis in the SU-DHL-4 cells, resulting in a notable cytotoxicity in B lymphocytes. (Supplementary Materials)

\section{References}

[1] N. Oishi and X. W. Wang, "Novel therapeutic strategies for targeting liver cancer stem cells," International Journal of Biological Sciences, vol. 7, no. 5, pp. 517-535, 2011.

[2] M. Nikolaou, A. Pavlopoulou, A. G. Georgakilas, and E. Kyrodimos, "The challenge of drug resistance in cancer treatment: a current overview," Clinical \& Experimental Metastasis, vol. 35, no. 4, pp. 309-318, 2018.

[3] M. Alev, L. Egenberger, L. Muhleisen et al., "Targeting of drugloaded nanoparticles to tumor sites increases cell death and release of danger signals," Journal of Controlled Release, vol. 285, pp. 67-80, 2018.

[4] M. N. Messmer, A. G. Snyder, and A. Oberst, "Comparing the effects of different cell death programs in tumor progression and immunotherapy," Cell Death and Differentiation, vol. 26, no. 1, pp. 115-129, 2019.

[5] M. B. Agarwal, "Is cancer chemotherapy dying?," Asian Journal of Transfusion Science, vol. 10, no. 3, pp. 1-7, 2016.

[6] N. R. Jabir, S. Tabrez, G. M. Ashraf, S. Shakil, G. A. Damanhouri, and M. A. Kamal, "Nanotechnology-based approaches in anticancer research," International Journal of Nanomedicine, vol. 7, pp. 4391-4408, 2012.

[7] S. A. Chew and S. Danti, "Biomaterial-based implantable devices for cancer therapy," Advanced Healthcare Materials, vol. 6, no. 2, 2017.

[8] S. Kim, Y. Shi, J. Y. Kim, K. Park, and J. X. Cheng, "Overcoming the barriers in micellar drug delivery: loading efficiency, in vivostability, and micelle-cell interaction," Expert Opinion on Drug Delivery, vol. 7, no. 1, pp. 49-62, 2010.

[9] C. von Roemeling, W. Jiang, C. K. Chan, I. L. Weissman, and B. Y. S. Kim, "Breaking down the barriers to precision cancer nanomedicine," Trends in Biotechnology, vol. 35, no. 2, pp. 159-171, 2017.

[10] C. D. Walkey, J. B. Olsen, H. Guo, A. Emili, and W. C. Chan, "Nanoparticle size and surface chemistry determine serum protein adsorption and macrophage uptake," Journal of the American Chemical Society, vol. 134, no. 4, pp. 2139-2147, 2012.

[11] J. Rueff and A. S. Rodrigues, "Cancer drug resistance: a brief overview from a genetic viewpoint," Methods in Molecular Biology, vol. 1395, pp. 1-18, 2016.

[12] B. Tyler, D. Gullotti, A. Mangraviti, T. Utsuki, and H. Brem, "Polylactic acid (PLA) controlled delivery carriers for biomedical applications," Advanced Drug Delivery Reviews, vol. 107, pp. 163-175, 2016.

[13] B. Layek, T. Sadhukha, J. Panyam, and S. Prabha, "Nanoengineered mesenchymal stem cells increase therapeutic efficacy of anticancer drug through true active tumor targeting," Molecular Cancer Therapeutics, vol. 17, no. 6, pp. 1196-1206, 2018.

[14] D. Rosenblum, N. Joshi, W. Tao, J. M. Karp, and D. Peer, "Progress and challenges towards targeted delivery of cancer therapeutics," Nature Communications, vol. 9, no. 1, p. 1410, 2018.

[15] L. Zhang, D. Jing, L. Wang et al., "Unique photochemoimmuno-nanoplatform against orthotopic xenograft oral cancer and metastatic syngeneic breast cancer," Nano Letters, vol. 18, no. 11, pp. 7092-7103, 2018.

[16] J. Ma, K. Kang, Y. Zhang, Q. Yi, and Z. Gu, "Detachable polyzwitterion coated ternary nanoparticles based on peptide dendritic carbon dots for efficient drug delivery in cancer therapy," ACS Applied Materials \& Interfaces, vol. 10, no. 50, pp. 4392343935, 2018.

[17] Z. Li, S. Tan, S. Li, Q. Shen, and K. Wang, "Cancer drug delivery in the nano era: an overview and perspectives (Review)," Oncology Reports, vol. 38, no. 2, pp. 611-624, 2017.

[18] A. Joly-Battaglini, C. Hammarstrom, B. Stankovic et al., "Rituximab efficiently depletes B cells in lung tumors and normal lung tissue," F1000Res, vol. 5, p. 38, 2016. 
[19] S. H. Lim and R. Levy, "Translational medicine in action: anti-CD20 therapy in lymphoma," Journal of Immunology, vol. 193, no. 4, pp. 1519-1524, 2014.

[20] M. G. Cipolleschi, I. Marzi, E. Rovida, M. Olivotto, and P. Dello Sbarba, "Low-dose methotrexate enhances cycling of highly anaplastic cancer cells," Cell Cycle, vol. 16, no. 3, pp. 280-285, 2017.

[21] N. Hagner and M. Joerger, "Cancer chemotherapy: targeting folic acid synthesis," Cancer Management and Research, vol. 2, pp. 293-301, 2010 


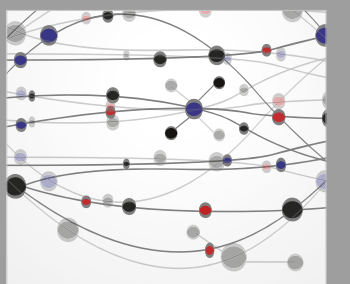

The Scientific World Journal
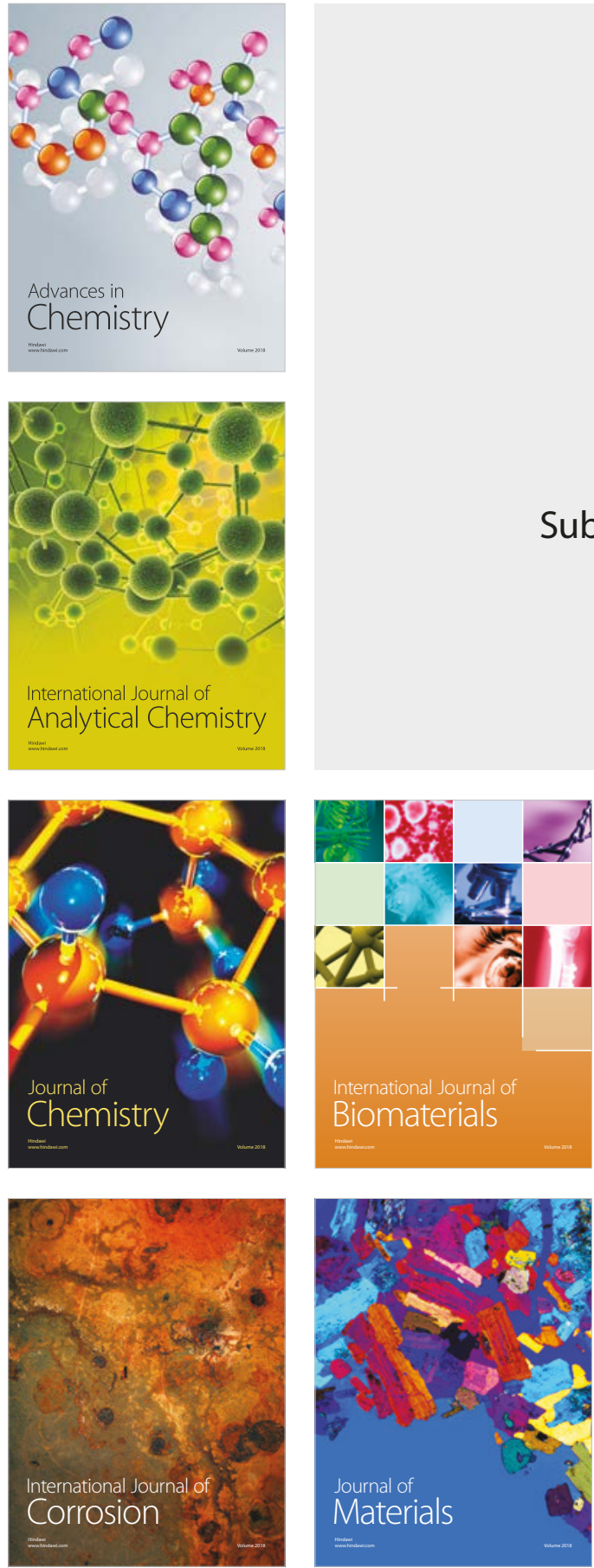

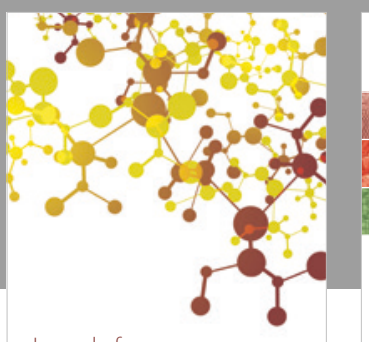

Journal of

Applied Chemistry
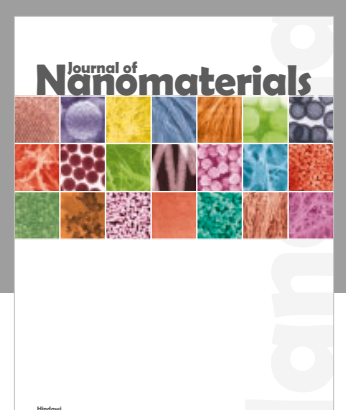

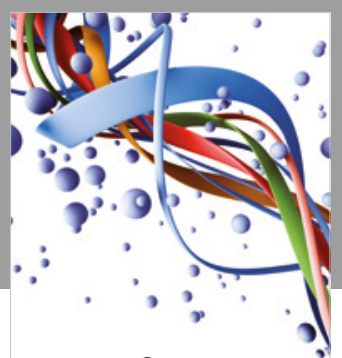

Scientifica

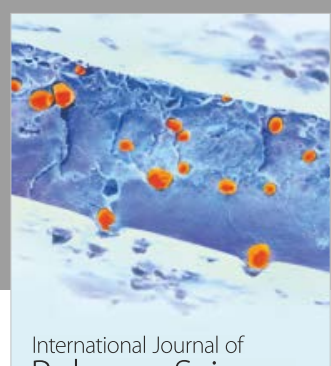

Polymer Science

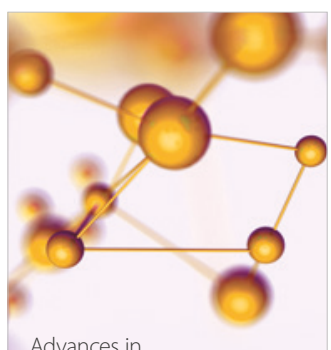

Physical Chemistry
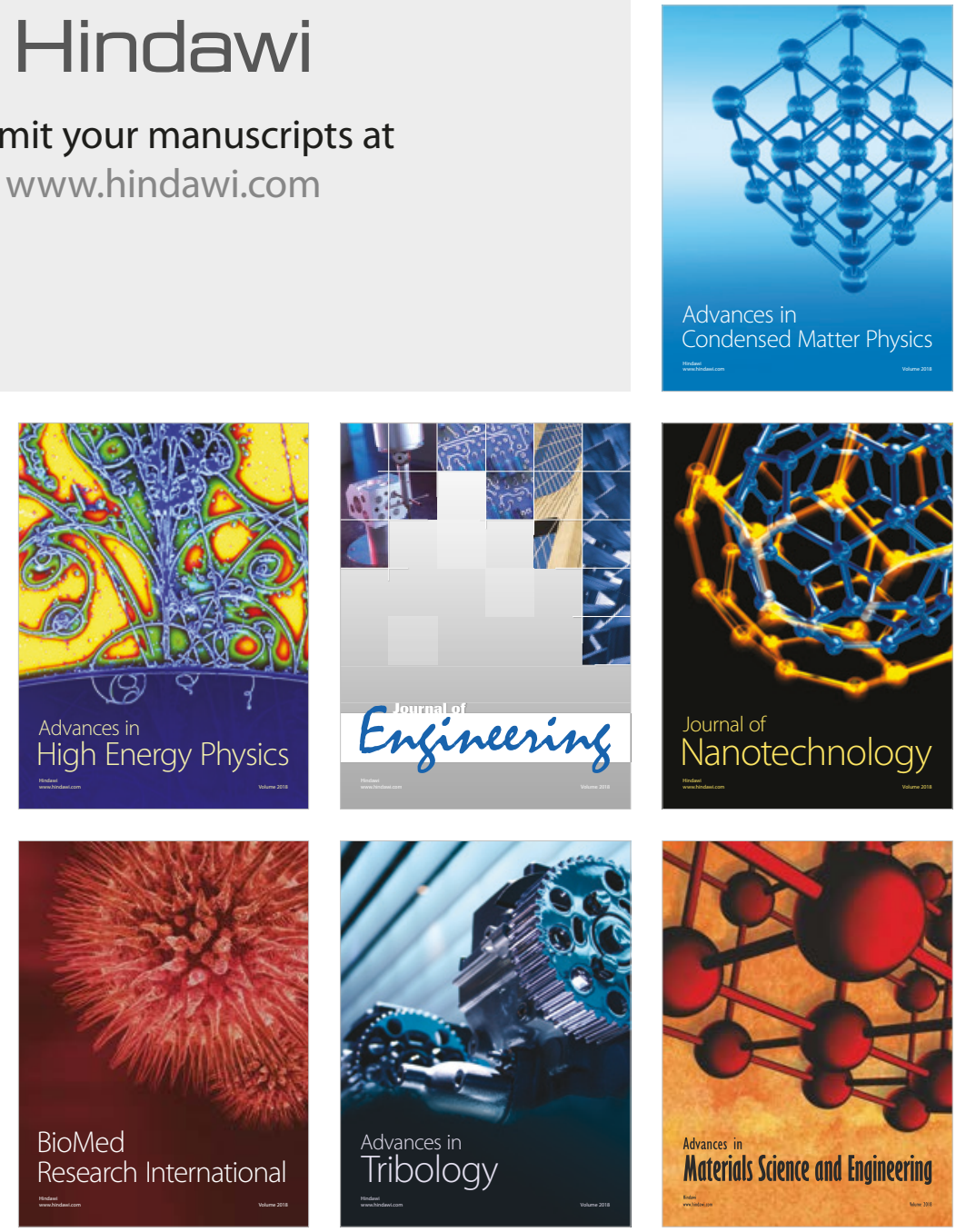